\title{
The fibronectin type-III (FNIII) domain of ATF7IP contributes to efficient transcriptional silencing mediated by the SETDB1 complex
}

\author{
Takeshi Tsusaka ${ }^{1,2}$, Kei Fukuda' ${ }^{1}$, Chikako Shimura' ${ }^{1}$, Masaki Kato ${ }^{1,3^{*}}$ and Yoichi Shinkai ${ }^{* *}$
}

\begin{abstract}
Background: The histone methyltransferase SETDB1 (also known as ESET) represses genes and various types of transposable elements, such as endogenous retroviruses (ERVs) and integrated exogenous retroviruses, through a deposition of trimethylation on lysine 9 of histone H3 (H3K9me3) in mouse embryonic stem cells (mESCs). ATF7IP (also known as MCAF1 or AM), a binding partner of SETDB1, regulates the nuclear localization and enzymatic activities of SETDB1 and plays a crucial role in SETDB1-mediated transcriptional silencing. In this study, we further dissected the ATF7IP function with its truncated mutants in Atf7ip knockout (KO) mESCs.

Results: We demonstrated that the SETDB1-interaction region within ATF7IP is essential for ATF7IP-dependent SETDB1 nuclear localization and silencing of both ERVs and integrated retroviral transgenes, whereas its C-terminal fibronectin type-III (FNIII) domain is dispensable for both these functions; rather, it has a role in efficient silencing mediated by the SETDB1 complex. Proteomic analysis identified a number of FNIII domain-interacting proteins, some of which have a consensus binding motif. We showed that one of the FNIII domain-binding proteins, ZMYM2, was involved in the efficient silencing of a transgene by ATF7IP. RNA-seq analysis of Atf7ip KO and WT or the FNIII domain mutant of ATF7IP-rescued Atf7ip KO mESCs showed that the FNIII domain mutant re-silenced most de-repressed SETDB1/ATF7IP-targeted ERVs compared to the WT. However, the silencing activity of the FNIII domain mutant was weaker than that of the ATF7IPWT, and some of the de-repressed germ cell-related genes in Atf7ip KO mESCs were not silenced by the FNIII domain mutant. Such germ cell-related genes are targeted and silenced by the MAX/MGA complex, and MGA was also identified as another potential binding molecule of the ATF7IP FNIII domain in the proteomic analysis. This suggests that the FNIII domain of ATF7IP acts as a binding hub of ATF7IP-interacting molecules possessing a specific interacting motif we named FAM and contributes to one layer of the SETDB1/ATF7IP complexmediated silencing mechanisms.
\end{abstract}

Conclusions: Our findings contributed to further understanding the function of ATF7IP in the SETDB1 complex, revealed the role of the FNIII domain of ATF7IP in transcriptional silencing, and suggested a potential underlying molecular mechanism for it.

Keywords: ATF7IP/AM/MCAF1, SETDB1/ESET, H3K9me3, FNIII domain, Transgene silencing, ZMYM2, MGA

*Correspondence: mkato@riken.jp; yshinkai@riken.jp

1 Cellular Memory Laboratory, RIKEN Cluster for Pioneering Research, Wako 351-0198, Japan

Full list of author information is available at the end of the article

\section{Background}

Gene expression patterns play a fundamental role in a variety of biological processes. In addition to the underlying DNA sequence and its methylation status, chromatin status also influences gene expression. Multiple chemical modifications occur in histone proteins, which are

(c) The Author(s) 2020. This article is licensed under a Creative Commons Attribution 4.0 International License, which permits use, sharing, adaptation, distribution and reproduction in any medium or format, as long as you give appropriate credit to the original author(s) and the source, provide a link to the Creative Commons licence, and indicate if changes were made. The images or other third party material in this article are included in the article's Creative Commons licence, unless indicated otherwise in a credit line to the material. If material is not included in the article's Creative Commons licence and your intended use is not permitted by statutory regulation or exceeds the permitted use, you will need to obtain permission directly from the copyright holder. To view a copy of this licence, visit http://creativeco mmons.org/licenses/by/4.0/. The Creative Commons Public Domain Dedication waiver (http://creativecommons.org/publicdomain/ zero/1.0/) applies to the data made available in this article, unless otherwise stated in a credit line to the data. 
the basic chromatin components, and they control multilayered chromatin structure and function. Methylation of lysine residues is a mark of both active and repressive gene expression, depending on the methylated residue, the methylation degree, and the chromatin region where the methylation happened [1]. Methylated histone $\mathrm{H} 3$ lysine 9 (H3K9) is associated with gene silencing, and it is dynamically regulated by several methyltransferases and demethylases. We have shown that in mESCs, one of the lysine methyltransferases, SETDB1 (also known as ESET) suppresses the expression of Class I and II endogenous retroviruses (ERVs) by depositing H3K9me3 marks [2, 3]. TRIM28 (also known as KAP1 or TIF1B) and TRIM28associated nucleic acid-binding zinc-finger proteins (ZFPs), including KRAB-ZFPs and YY1, play an important role in SETDB1 targeting to ERVs and silencing them [4-6]. In addition, the recent studies in human cells have shown that the human silencing hub (HUSH) complex, comprised MPP8, FAM208A (also known as TASOR), and Periphilin, recruits SETDB1 to a transgene that is integrated into heterochromatin to induce H3K9me3mediated silencing of its expression [7]. However, differences between the two silencing mechanisms have not yet been fully clarified.

ATF7IP (also known as AM or MCAF1) is a binding partner of SETDB1 [8, 9]. Loss of ATF7IP results in the de-repression of SETDB1-regulated genes, ERVs, and the transgene integrated by retroviruses, concomitant with a decreased H3K9me3, which are similar to that conferred by SETDB1 inactivation, but these phenotypes are weaker than those observed in SETDB1 inactivation [10-15]. Recently, we reported one role of ATF7IP in SETDB1, in which ATF7IP regulates SETDB1 nuclear localization and increased levels of its ubiquitinated and more enzymatically active forms [16]. However, given that SETDB1 can be imported into the nucleus and recruited to the target loci without ATF7IP [16, 17], the residual SETDB1 in the nucleus could execute a large part of its silencing function even in the absence of ATF7IP, which is consistent with the weaker phenotype of the loss of ATF7IP. Prior studies identified two functional regions within human ATF7IP: SETDB1- and MBD1-binding regions [9, 18]. Since the MBD1-binding region is also characterized as a fibronectin type-III (FNIII) domain, we referred to the region hereinafter as the "FNIII domain". Although the residues 562-817 within human ATF7IP were shown to be essential for binding to SETDB1 [9], the functional requirement of this binding region has not been studied yet. The FNIII domain was shown to be essential for the binding of ATF7IP to MBD1, and this interaction is mediated by residues 529-592 of human MBD1, which is included in the transcriptional repression domain (TRD) of MBD1 [18]. Substitutions with arginine (R) at residues
I576 and L579 of human MBD1 disrupted its interaction with human ATF7IP as well as its transcriptional repression [9, 19-21]. To further understand the function of ATF7IP in transcriptional silencing, we generated a SETDB1-binding domain and FNIII domain deletion mutant of mouse ATF7IP and performed rescue experiments of our established Atf7ip KO mESCs with these two deletion mutants.

\section{Results \\ Distinct functional requirements of the SETDB1-binding region and the FNIII domain in ATF7IP-dependent retroelement silencing}

We have previously established Atf7ip KO cells using mESCs infected with the murine stem cell virus (MSCV) carrying the GFP gene as a background [22] and observed that the Atf7ip KO ESCs showed increased expression of SETDB1-regulated ERVs and the MSCV-GFP reporter [16]. For rescue experiments with ATF7IP, we used a piggyBac transposase-based vector for the expression of 3xFLAG-tagged mouse ATF7IP with either WT or each domain's deletion mutants: dSETDB1 lacking residues 627-694, which is within the corresponding SETDB1 binding domain of mouse ATF7IP and covering two estimated $\alpha$-helix regions, and dFNIII lacking residues 1190-1306 of the FNIII domain, which is highly conserved between human and mouse ATF7IP (Fig. 1a and Additional file 1: Fig. S1). These piggyBac transposasebased vectors can be integrated into the host genome and stably express exogenous FLAG-tagged ATF7IPs. By the transfection of each plasmid and continuous drug selection, we established Atf7ip KO mESCs expressing 3xFLAG-ATF7IP-WT, dSETDB1, or dFNIII mutants and performed a co-immunoprecipitation (co-IP) assay with these cell lines using a FLAG M2 affinity gel. As expected, the dSETDB1 mutant could not co-IP endogenous SETDB1, while the WT and the dFNIII mutant could (Fig. 1b). We then examined the RNA expression of exogenous MSCV-GFP and ERVs regulated by ATF7IP and SETDB1 under long-term culturing conditions (greater than 2 weeks). As shown in Fig. 1c, the ATF7IP WT and the dFNIII mutant re-silenced the de-repressed MSCVGFP and ERV expression, while the dSETDB1 mutant did not, suggesting that its interaction with SETDB1 is essential for the role of ATF7IP in transcriptional silencing.

We next examined the silencing kinetics of these rescued cells by taking advantage of the MSCV-GFP reporter (Fig. 1d). We transfected 3xFLAG-ATF7IP with either WT, dSETDB1, or dFNIII expression vectors into Atf7ip KO mESCs, and the transfected cells were selected by continuous puromycin treatment. At day 5 after transfection, GFP expression levels were analyzed by flow cytometry. As expected, the 


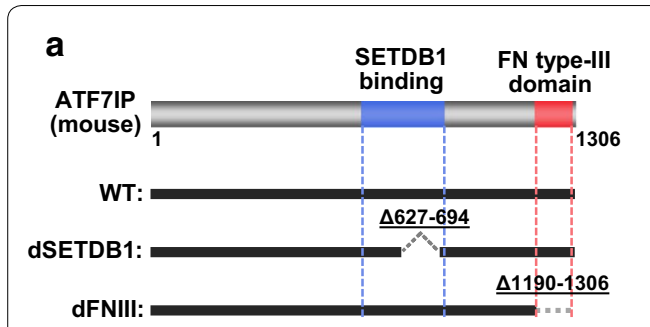

b

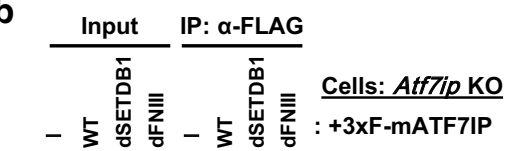

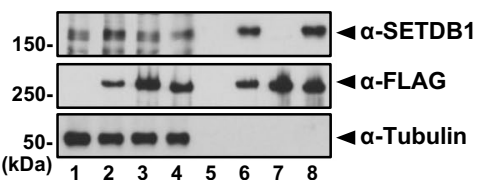

d
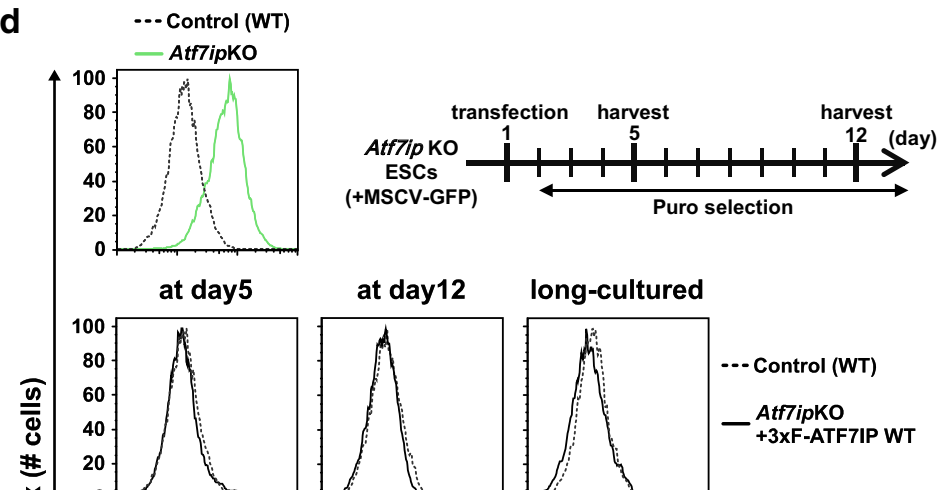

at day12
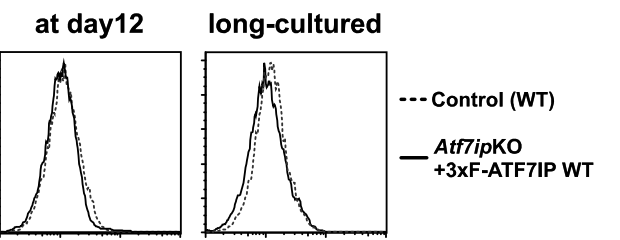

e
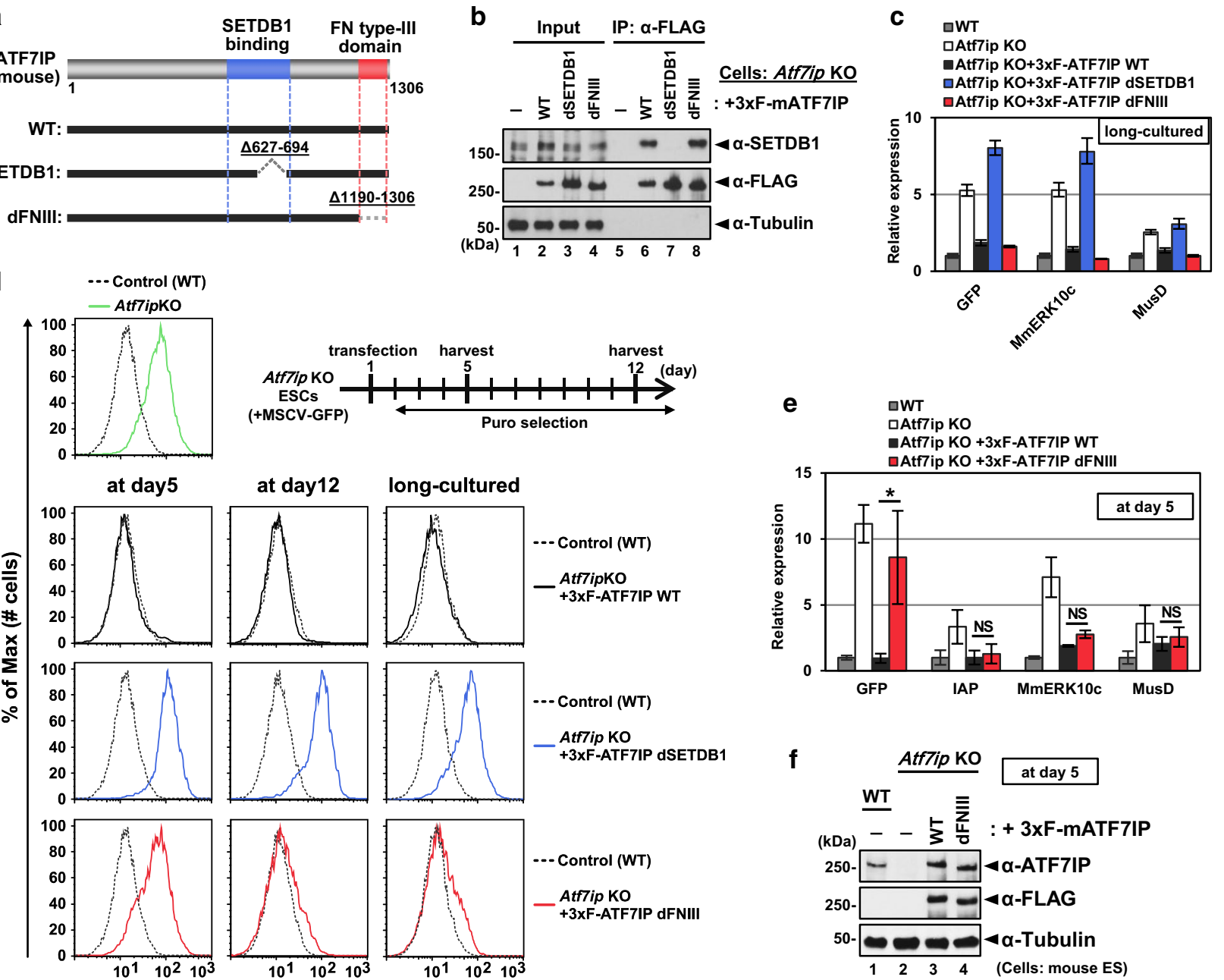

...-Control (WT)

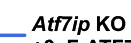

+3XF-ATF7IP dSETDB1

f

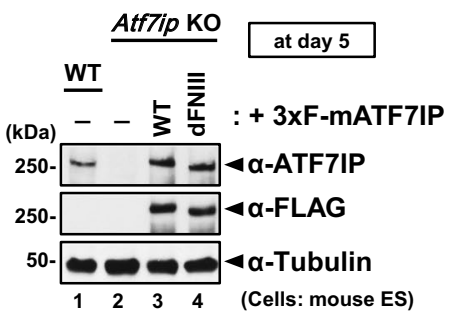

Fig. 1 Molecular mapping analysis of ATF7IP in mESCs. a Domain architecture of mouse ATF7IP protein. Deletion mutants used in this study are shown. $\mathbf{b}$ Interaction of SETDB1 with each 3xFLAG-ATF7IP expressed in Atf7ip KO mESCs. Co-IP assay was performed with the cell lines using anti-FLAG and shows that the WT and the dFNIII mutant, but not the dSETDB1 mutant, bound to SETDB1. c RT-qPCR analysis was performed with long-cultured cells after the transfection. RNA expression was normalized to Hprt expression and is shown relative to the level in WT cells. Data are mean \pm SD; $n=3$, technical replicates. $\mathbf{d}$ MSCV-GFP expression was analyzed by flow cytometric analysis at indicated time points. The transfected cells were subjected to continuous drug selection from 1 day after the transfection. e RT-qPCR analysis was performed with samples collected at day 5. RNA expression was normalized to Hprt expression and is shown relative to the level in WT cells. Data are mean $\pm S E M ; n=4$ without IAP $(n=3)$ from four or three experiments. NS: $P>0.05,{ }^{*} P<0.05$ by unpaired Student's t-test. $\mathbf{f}$ WB analysis was performed with samples collected at day 5. Comparative expression between the WT and the dFNIII mutant ATF7IP was confirmed

WT-rescued cells showed low GFP expression, similar to that of the parental Atf7ip WT cells, and the dSETDB1-rescued cells showed higher GFP expression, as seen in the Atf7ip KO cells. Unexpectedly, the expression of the dFNIII mutant could not re-silence MSCV-GFP expression on day 5. However, the higher GFP expression in the dFNIII-expressing cells was repressed to the WT level after culturing them for an additional seven days (at day 12). Consistent with this observation, the dFNIII-rescued cells at day 5 showed higher expression of GFP mRNA as compared to that of the WT-rescued cells (Fig. 1e). We then confirmed the expression of exogenous ATF7IP protein between the WT- and dFNIII-rescued cells at day 5 by western blot analysis (Fig. 1f). Interestingly, ERVs were re-silenced by the expression of the dFNIII mutant, similar to WT expression, even at day 5 (Fig. 1e). These results suggest that the FNIII domain plays a role in ATF7IP-mediated transcriptional silencing under certain condition. 
Interaction with SETDB1, but not the FNIII domain of ATF7IP, is required for ATF7IP-dependent SETDB1 nuclear localization

We have recently reported that ATF7IP regulates SETDB1's nuclear localization by antagonizing and enhancing its nuclear export and import, respectively [16]. Therefore, we determined whether the expression of the dSETDB1 or FNIII mutant can rescue the cytoplasmic accumulation phenotype of SETDB1 in Atf7ip KO mESCs. We examined SETDB1 localization in the longcultured 3xFLAG-tagged ATF7IP WT-, dSETDB1-, and dFNIII-rescued cells by immunofluorescence (IF) analysis. The WT and the two mutants of 3xFLAG-tagged ATF7IP were all localized in the nucleus (Fig. 2a; quantification in Fig. 2b, right). The expression of the ATF7IP WT and the dFNIII mutant in Atf7ip KO cells restored the SETDB1's nuclear localization (Fig. 2a; quantification in Fig. 2b, left) without significant changes in nuclear foci numbers (Fig. 2c, d, left). Both the ATF7IP WT and the dFNIII mutant also co-localized with SETDB1 in the nucleus and in the nuclear foci (Fig. 2a). In contrast, the dSETDB1 mutant could not rescue the cytoplasmic localization of SETDB1 (Fig. 2a; quantification in Fig. 2b, left), and the number of dSETDB1 nuclear foci was reduced (Fig. 2a; quantification in Fig. 2c, d, right). The dFNIIIrescued cells also showed that SETDB1 and exogenous ATF7IP were localized in the nucleus as efficiently as those in the ATF7IP WT-rescued Atf7ip KO cells five days after transfection (Additional file 2: Fig. S2A-C). These results suggest that the regulation of SETDB1 nuclear localization by ATF7IP requires their interaction a

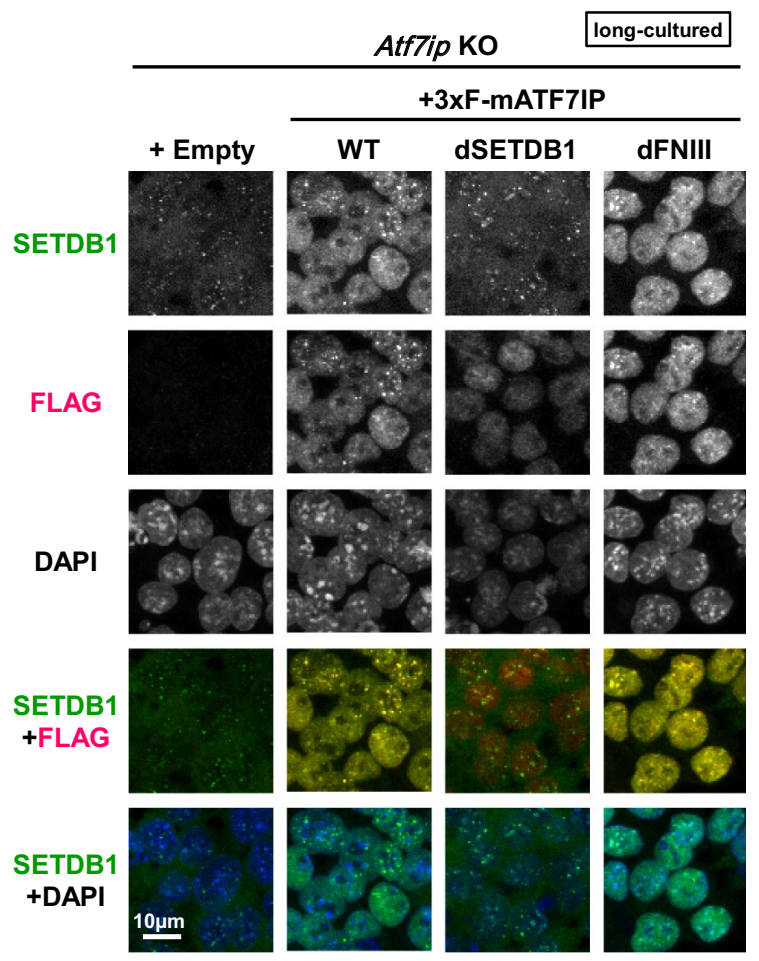

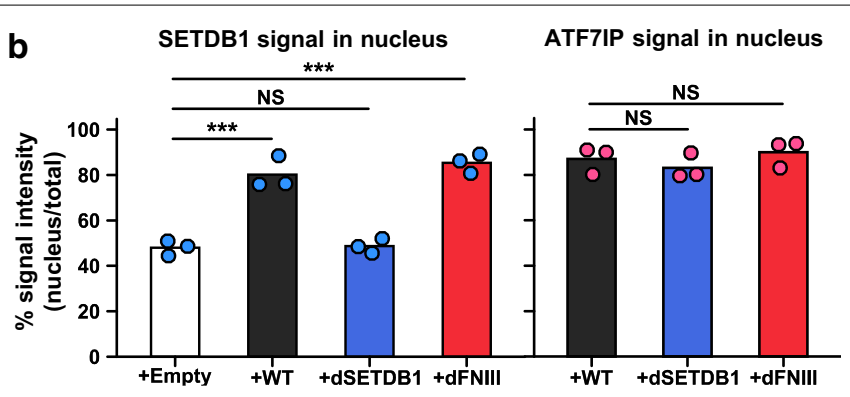

C SETDB1 foci (Total) ATF7IP foci (Total)

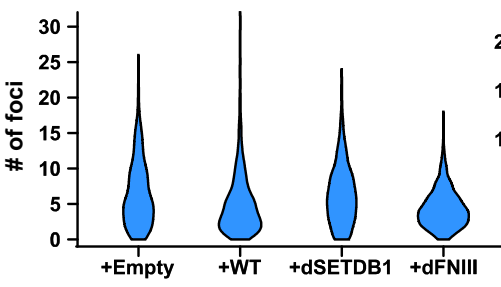

d

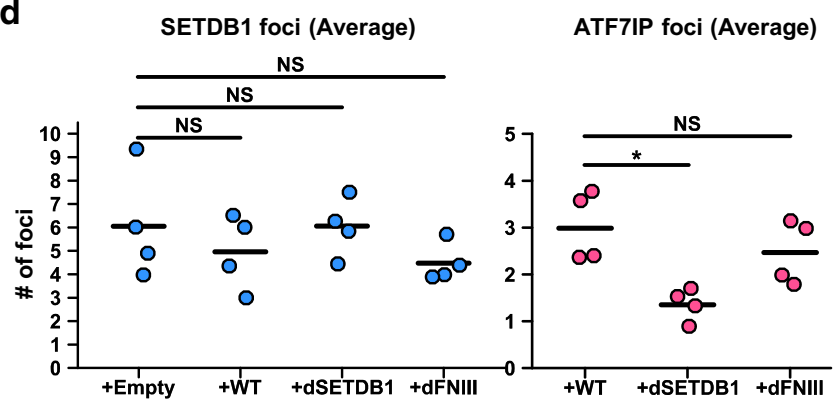

Fig. 2 The SETDB1-binding region, but not the FNIII domain, within ATF7IP is required for normal localization of SETDB1. a IF analysis shows that exogenous expression of 3xFLAG-ATF7IP WT and dFNIII mutant, but not dSETDB1 mutant, rescues the abnormal localization of SETDB1 in Atf7ip KO mESCs. Representative images are shown, and the quantitative analyses are shown in F-H. Scale bar: $10 \mu \mathrm{m}$. b SETDB1 (left) and 3xFLAG-ATF7IP (right) signals in the nucleus that was determined by DAPI staining were calculated. The mean from three independent experiments is shown as a bar graph with jittered points indicating the average \% intensity of each experiment. Over 100 cells were analyzed per sample per experiment. NS (not significant): $P>0.05,{ }^{* * *} P<0.001$ versus " + Empty" group by Dunnett's test. c, $\mathbf{d}$ SETDB1 (left) and 3xFLAG-ATF7IP (right) foci in the nucleus that was determined by DAPI staining were calculated. Violin plot for their foci numbers from all the cells analyzed are shown (c). The mean from four independent experiments is shown as a bar with jittered points indicating the average number of each experiment (d) 
and that a delayed silencing of MSCV-GFP in dFNIII mutant-rescued cells does not seem to be caused by SETDB1's delayed nuclear localization or mislocalization.

\section{Identification of ATF7IP FNIII domain-binding proteins}

We further sought to reveal the underlying mechanism for the inefficient re-silencing of the MSCV-GFP reporter transgene by the dFNIII mutant (Fig. 1d). Since the FNIII domain functions as a binding domain for MBD1 in human cell lines $[9,18]$, we searched for binding proteins for the FNIII domain of ATF7IP in mESCs. For this, we performed a proteomic analysis with the recombinant FNIII domain of mouse ATF7IP and nuclear lysates of mESCs (Fig. 3a). The nuclear fractions from Atf7ip KO mESCs were incubated with a GST-tagged FNIII domain, produced in, and purified from E. coli. After purification with glutathione beads, the bound proteins with the FNIII domain were identified by liquid chromatography followed by tandem mass spectrometry (LC-MS/ MS) analysis (Fig. 3b, the full list is in Additional file 3: Table S1). We identified over 20 proteins enriched in the FNIII domain-pulled-down sample, with a high coverage, and found some known protein networks, including a ZMYM2 (also known as ZNF198)-LSD1 (also known as KDM1A)-HDAC complex [23, 24], by STRING analysis (Additional file 4: Fig. S3). Unexpectedly, we could not recover MBD1 in our proteomic analysis, suggesting that MBD1 may not exist in Atf7ip KO mESCs or that their interaction may not occur significantly in mESCs. Using a co-IP experiment with transient ectopic expression in HEK293T cells, we validated the interaction of full-length ATF7IP with several high-ranked candidates, including ZMYM2, MGA (residues 2362-3003), ZFP518A, and KIAA1551, and a known interactor MBD1 (Fig. 3c). As expected, the dFNIII mutant could not bind to these proteins, but it could bind to SETDB1 (Fig. 3d), supporting the hypothesis that these newly identified interactors, as well as MBD1, can bind to the FNIII domain of ATF7IP.

To examine which region of ZMYM2, which is a topranked protein in our proteomic analysis, is essential for its interaction with ATF7IP, we performed co-IP experiments with a series of truncated mutants of ZMYM2 using HEK293T cells (Additional file 5: Fig. S4A-D). These results suggest that residues 181-350 within ZMYM2 seem to be important for its interaction with ATF7IP. Visual inspection of this region revealed that it contains two sequences (referred to as FNIII domain of ATF7IP-interacting motif 1 (FAM1) and FAM2, explained later) similar to an "ITEFSL" sequence within the TRD of MBD1, which was shown to be essential for its binding to the FNIII domain [18]. As substitutions of isoleucine (I) and leucine (L) to arginine (R) within this sequence perturbed the interaction between MBD1 and the FNIII domain $[9,18]$, we wondered whether similar mutations at the corresponding "V" and "L" residues of FAM1 and/or FAM2 within ZMYM2 can affect the interaction between ZMYM2 and ATF7IP (see Additional file 6: Fig. S5A). We transfected 3xFLAG-ATF7IP with either the control empty vector, V5-ZMYM2-WT, FAM1 mutant (V187R/L190R), FAM2 mutant (V217R/L220R), FAM1 and FAM2 double mutant, or a d39aa mutant that lacks residues 182-220 into HEK293T cells and performed co-IP experiments with an anti-FLAG M2 affinity gel (Fig. 3e). The results showed that the mutations at either FAM1 or FAM2 impaired the interaction, and that the FAM1 and 2 mutant or the d39aa mutant completely failed to co-IP ATF7IP, suggesting that both FAM1 and FAM2 contribute to the binding with ATF7IP. We further examined the interaction of ZMYM2 with ATF7IP using a GST pull-down assay (Fig. 3f). The HEK293Tcell lysates transfected with V5-ZMYM2 WT or FAM1\&2 Mut were pulled-down with a recombinant GST or a recombinant GST-FNIII domain. Western blot analysis showed that the GST-FNIII domain, but not GST alone, bound to ZMYM2 WT, and that the binding of the FAM1 and 2 mutant to ATF7IP was severely impaired compared to that of the ZMYM2 WT. These data suggest that ZMYM2 binds to the FNIII domain of ATF7IP via its FAM1 and FAM2 motifs. We then examined primary sequences of the identified FNIII-binding proteins and found that MGA, ZMYM4, and ZFP518A possess an "ITEFSL"-like sequence (Fig. 3g). We showed that mutations on the motif abolished the interaction of those proteins with ATF7IP (Additional file 6: Fig. S5A-C). Therefore, we proposed that the "ITEFSL"-like sequences are a consensus binding motif for the FNIII domain of ATF7IP, and referred to as FAM.

\section{ZMYM2 is involved in the efficient silencing of exogenous provirus reporter by ATF7IP}

Among the identified binding proteins for the FNIII domain of ATF7IP, we focused our attention on the top-ranked protein ZMYM2, which has two FAMs, and may function with the LSD-HDAC1 repressor as a complex [23, 24]. We established Zmym2 KO mESCs using CRISPR/Cas9 technology. mESCs harboring the hCas9 and MSCV-GFP reporter were transfected with an expression vector for gRNA targeting the mouse Zmym2 gene. We observed a slight increase in the MSCV-GFP reporter in the Zmym2-gRNA-transfected cells by flow cytometry analysis (Additional file 7: Fig. S6A) and sorted the cell populations with high GFP intensity. The sorted cells were cloned, and the ZMYM2 expression in the cloned cell lines were subsequently analyzed. We finally isolated two independent clones of Zmym2 KO mESCs, as evidenced by western blot using 


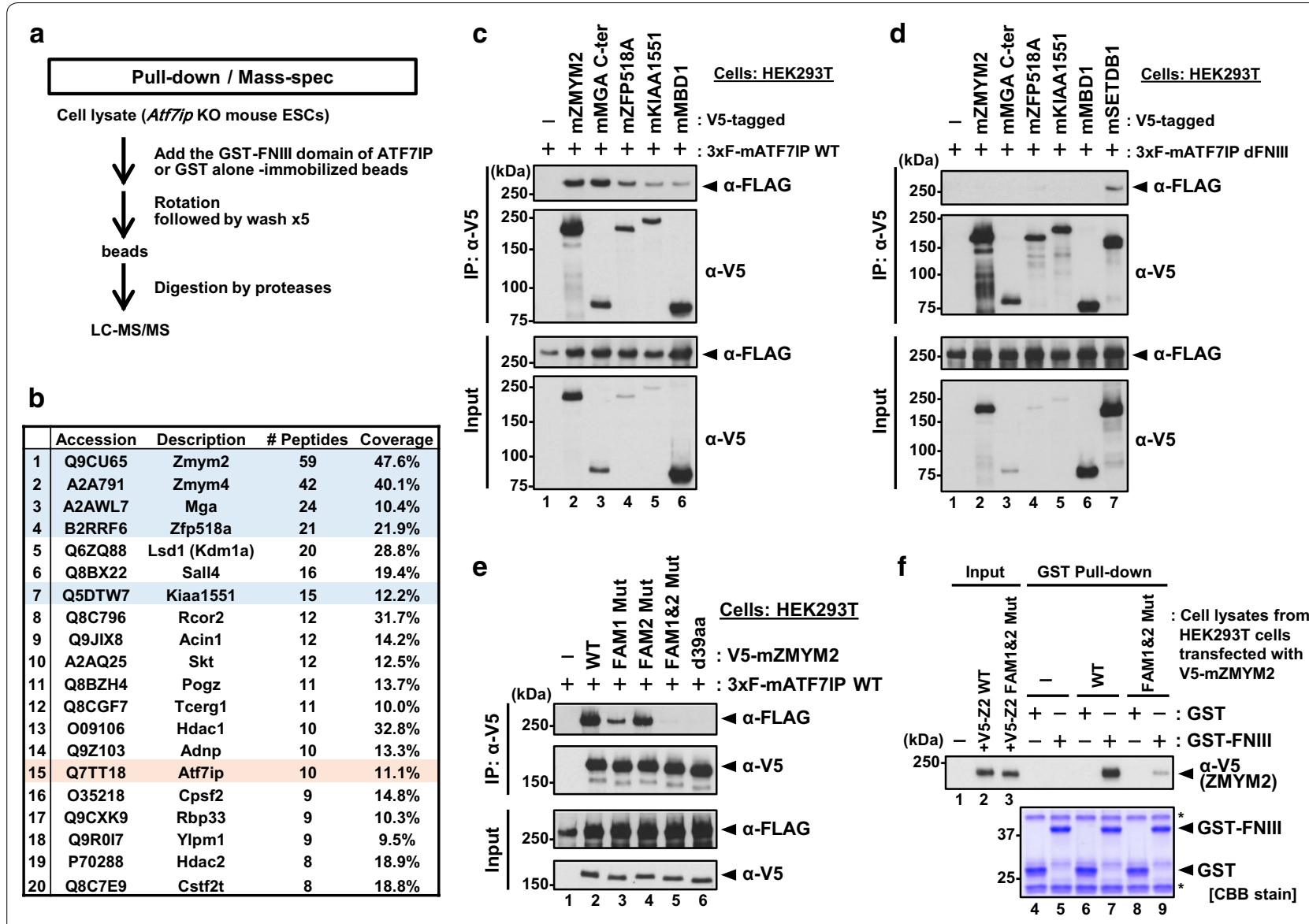

g

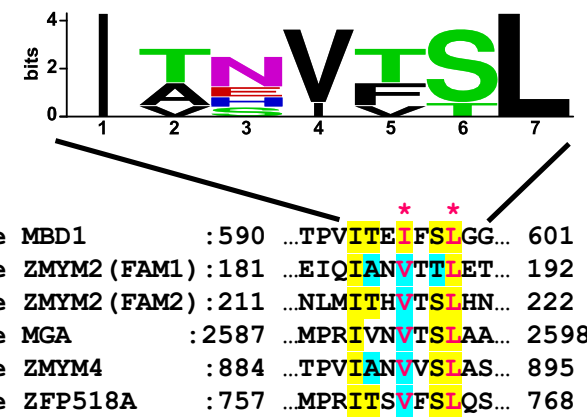

FAM: FNIII domain of ATF7IP -interacting Motif

Fig. 3 Identification of binding proteins for ATF7IP's C-terminal FNIII domain. a An experimental design for identification of binding proteins of ATF7IP's FNIII domain by pull-down assay followed by LC-MS/MS analysis. b A list of top 20 proteins identified in the proteomic analysis. The proteins which was confirmed to interact with ATF7IP by independent experiment are filled in blue. ATF7IP is filled in red. c Co-IP assay performed with HEK293T cells transiently co-transfected with 3xFLAG-tagged ATF7IP WT and V5-tagged proteins. MBD1, a known ATF7IP interactor, was used as a positive control. All the high-ranked proteins bound to ATF7IP. $\mathbf{d}$ Co-IP assay performed with HEK29T cells transiently co-transfected with 3xFLAG-tagged ATF7IP dFNIII mutant and V5-tagged indicated proteins plus SETDB1. The FNIII mutant bound to SETDB1, but not to other proteins. e Co-IP assay performed with HEK29Tcells transiently co-transfected with 3xFLAG-tagged ATF7IP WT and V5-tagged ZMYM2 WT or mutants. FAM1 Mut possesses substitutions, V187R and L190R; FAM2 Mut possesses substitutions V217R and L220R; FAM1\&FAM2 Mut possesses all the four substitutions; $\triangle 39$ aa mutant lacks the region of 182-220 amino acids of ZMYM2. $\mathbf{f} F$ FNIII domain of ATF7IP is sufficient for the interaction with ZMYM2. GST-pull down assay with recombinant FNIII domain of ATF7IP and cells lysates from HEK293T cells transiently transfected with V5-ZMYM2 WT or FAM1\&2 Mut was performed. The pulled-down samples were analyzed by WB. $\mathbf{g}$ Alignment of MBD1-resembled sequences within the indicated proteins and their sequence logo. Asterisk indicates important residues for the interaction with ATF7IP. The substitutions of the I/N and $L$ with $R$ disrupted the interaction with ATF7IP. This consensus sequence is proposed as a FN III domain of ATF7IP-interacting Motif (FAM). The sequence logo was created by WEBLOGO (version 2.8.2) 
an anti-ZMYM2 antibody (Fig. 4a). We found that both established Zmym2 KO cell lines showed GFP expression equivalent to the parental WT cells (Additional file 7: Fig. S6B), resembling the case of the ATF7IP FNIII-rescued
Atf7ip KO mESCs after long-term culture (Fig. 1d). We then transfected Zmym2 KO cells with a 3xFLAGtagged ZMYM2 WT expression vector and confirmed their expression (Fig. 4a). By using the 3xFLAG-tagged

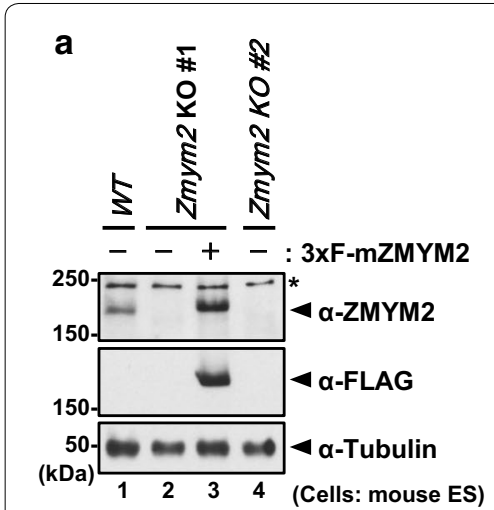

b

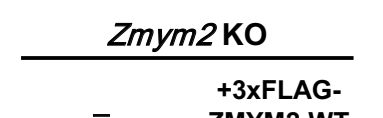

FLAG

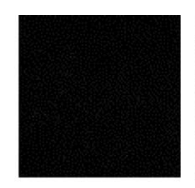

ZMYM2 WT

SETDB1

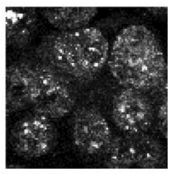

DAPI

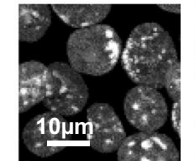

SETDB1

+FLAG
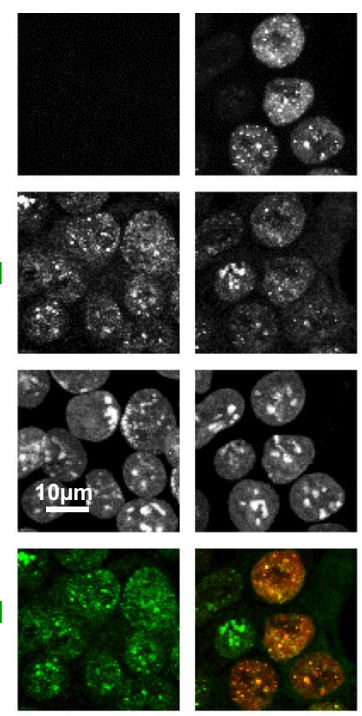

e
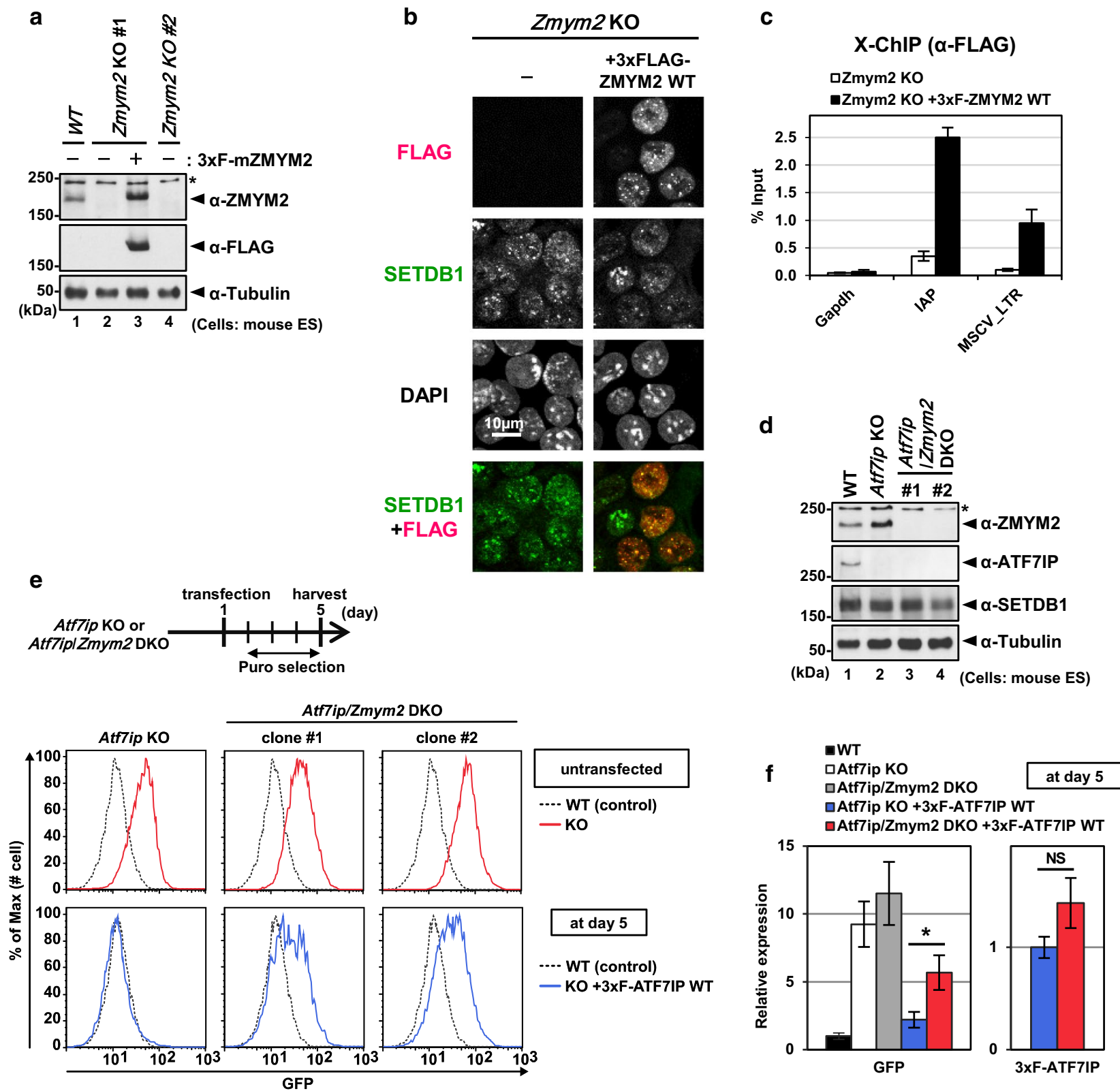

Fig. 4 ZMYM2 partly mediates efficient re-silencing of MSCV-GFP reporter by ATF7IP. a WB analysis confirms no expression of ZMYM2 in Zmym2 $\mathrm{KO}$ cell lines and shows an exogenous expression of 3xFLAG-ZMYM2. b IF analysis shows the co-localization of 3xFLAG-ZMYM2 with SETDB1's nuclear foci. c X-ChIP analysis with anti-FLAG antibody at the indicated genomic loci in Zmym2 KO mESCs and the Zmym2 KO cells rescued by 3XFLAG-ZMYM2. Gapdh gene was used as a negative control. The ChIP enrichment levels are shown as mean $\pm S E M ; n=3$ from three experiments. d WB analysis confirms no expression of both ATF7IP and ZMYM2 in Atf7ip/Zmym2 DKO cells. e MSCV-GFP expression was analyzed by flow cytometric analysis at day 5 after the transfection. Atf7ip/Zmym2 DKO cells show higher expression of GFP, compared to the parental WT cells. f RT-qPCR analysis was performed with samples collected at day 5 after the transfection. RNA expression was normalized to Hprt expression and is shown relative to the level in WT cells (left) or Atf7ip KO cells transfected with 3xFLAG-ATF7IPWT. Data are mean \pm SEM; $n=4$ from four experiments. NS: $P>0.05,{ }^{*} P<0.05$ by unpaired Student's $t$ test 
ZMYM2-rescued cells, we observed the co-localization of 3xFLAG-ZMYM2 with endogenous SETDB1 at the foci in the nucleus by IF analysis (Fig. 4b), suggesting a potential function of ZMYM2 in interacting with SETDB1 and ATF7IP. Furthermore, we found an enrichment of the FLAG-tagged ZMYM2 at the SETDB1/ ATF7IP-target genomic regions, including the LTR of MSCV-GFP (Fig. 4c).

To examine the potential involvement of ZMYM2 in the re-silencing of the MSCV-GFP reporter by ATF7IP, we further inactivated Zmym2 in Atf7ip KO mESCs. We confirmed the depletion of ZMYM2 protein in the two Zmym2/Atf7ip DKO cell lines by western blot analysis (Fig. 4d). Furthermore, we found an upregulation of ZMYM2 protein in Atf7ip KO mESCs as compared to the parental WT cells (Fig. 4d), suggesting the existence of a potential negative feedback mechanism. We then transfected either Atf7ip KO cells or Zmym2/Atf7ip DKO cell lines with 3xFLAG-ATF7IP WT and analyzed them by flow cytometric analysis five days after transfection. We found that the re-expression of ATF7IP WT in the Zmym2/Atf7ip DKO cell lines incompletely silenced the expression of MSCV-GFP reporter (Fig. 4e). Consistent with this, we observed, by RT-qPCR analysis that although the expression levels of 3xFLAG-ATF7IP WT mRNA were similar, the 3xFLAG-ATF7IP WTrescued Atf7ip/Zmym2 DKO cells showed $\sim$ threefold increase in the expression of GFP mRNA as compared to the 3xFLAG-ATF7IP WT-rescued Atf7ip KO cells at day 5 after transfection (Fig. 4f). Taken together, these results suggest that ZMYM2 partly mediates the efficient re-silencing of the MSCV-GFP reporter by ATF7IP in mESCs.

\section{The FNIII domain of ATF7IP contributes to the efficient silencing of SETDB1 target ERVs and some MGA/ MAX-targeted germ cell-related genes}

To further elucidate the role of the FNIII domain in ATF7IP-mediated transcriptional regulation, we performed RNA-seq analysis of WT, Atf7ip KO, and Atf7ip KO stably rescued with WT or FNIII domain mutant of ATF7IP and Zmym2 KO mESCs (Additional file 8: Fig.
S7). In comparison with parental WT ESCs, 87 and 69 genes were commonly up- and downregulated, respectively, $(\mathrm{FDR}<0.05, \mathrm{FC} \geq 2)$ in two independent Atf7ip KO mESC clones, TT\#2-5 and TT\#2-12 [16] (Fig. 5a and Additional file 9: Table. S2). A majority of the upregulated genes (76/87) in Atf7ip KO cells were repressed by exogenous ATF7IP WT expression (Fig. 5b). Complementation with ATF7IP WT induced a greater number of up- or downregulated genes compared to the Atf7IP KO (Fig. 5a). When the FNIII domain mutant was introduced into Atf7ip KO ESCs, a majority of the upregulated genes $(70 / 87)$ in Atf7ip KO mESCs were reversed to WT levels (Fig. 5b). Interestingly, Gene Ontology (GO) term enrichment analysis using DAVID 6.7 [25] showed that GO terms related to the meiotic cell cycle or spermatogenesis were enriched in the 12 genes that were re-silenced in the WT rescued cells, but not in the dFNIII mutant-rescued cells (Fig. 5c). These include Rec114, Tex11, Tex15, Fkbp6, Sycp1, Stra8, and Mael (Additional file 9: Table S2). Such germ cell-related genes were also de-repressed in Setdb1 $\mathrm{KO}$ mESCs [3]. Furthermore, it has been reported that these genes were also induced in the Max knockdown (KD) mESCs, and that some of them were de-repressed in Atf7ip KD mESCs [26]. RT-qPCR analysis confirmed that the de-repressed germ cell-related genes were not repressed by the FNIII domain mutant in Atf7ip KO ESCs at day 5 after transfection or were only partially silenced over longer culture conditions, whereas these genes were efficiently silenced by ATF7IP WT even at day 5 posttransfection (Fig. 5d). Thus, the FNIII domain has an indispensable role in the ATF7IP-mediated silencing of some MAX-regulated germ cell-related genes.

In the case of the retroelements, 10 different classes of repeats were upregulated in Atf7ip KO mESCs, which was consistent with previous findings [15], and all of them were repressed by exogenous ATF7IP WT expression (Fig. 5a, e). The introduction of the FNIII domain mutant also repressed most of the de-repressed repeats (9/10) (Fig. 5a, e). Interestingly, the other 10 different classes of retroelements were further downregulated in Atf7ip KO mESCs rescued with ATF7IP WT (Fig. 5a, f). The additionally downregulated retroelements were

\footnotetext{
(See figure on next page.)

Fig. 5 FNIII domain of ATF7IP contributes to efficient silencing of SETDB1 target ERVs and some of MGA/MAX target germ cell-related genes. a The number of DE genes and repeats (FDR $<0.05, F C \geq 2$ ) in Atf7ip KO, Atf7ip KO rescued with WT or the FNIII domain mutant of ATF7IP and Zmym2 KO ESCs. b Overlap of differentially upregulated genes in Atf7ip KO ESCS, Atf7ip KO ESCs rescued with WT or the FNIII domain mutant of Atf7ip. c GO term enrichment analysis for cellular component of up-regulated genes in Atf7ip KO ESCs (upper panel) or commonly upregulated genes in Atf7ip KO and Atf7ip KO ESCs rescued with the FNIII domain mutant of ATF7IP (lower panel). The analysis was performed by DAVID. $\mathbf{d}$ RT-qPCR analysis of germ-cell related genes in WT, Atf7ip KO, Atf7ip KO ESCs rescued with WT or the FNIII domain mutant of ATF7IP. RNA samples were collected at day 5 (left) or more than 2 weeks (right) after the transfection. RNA expression was normalized to Hprt expression and is shown relative to the level in WT cells. Data are mean \pm SEM; $n=4$. NS: $P>0.05,{ }^{*} P<0.05$ by unpaired Student's $t$ test. e Up-regulated repeats in Atf7ip KO mESCs. f Down-regulated repeats in Atf7ip KO mESCs expressing Atf7ip WT transgene. $\mathbf{g}$ Overlap of 3xFLAG-ATF7IP WT and FNIII domain mutant stringent peaks. $\mathbf{h}$ Enrichment of FLAG-ATF7IP in retroelements
} 


a
\begin{tabular}{|c|c|c|c|c|}
\hline Gene & Atf7ip KO & $\begin{array}{c}\text { Atf7ip KO } \\
+ \text { WT }\end{array}$ & $\begin{array}{c}\text { Atf7ip KO } \\
+ \text { dFNIII }\end{array}$ & Zmym2 KO \\
\hline Up & 87 & 278 & 36 & 178 \\
\hline Down & 69 & 293 & 51 & 366 \\
\hline Repeat & Atf7ip KO & $\begin{array}{c}\text { Atf7ip KO } \\
+ \text { WT }\end{array}$ & $\begin{array}{c}\text { Atf7ip KO } \\
+ \text { dFNIII }\end{array}$ & Zmym2 KO \\
\hline Up & 10 & 0 & 1 & 6 \\
\hline Down & 0 & 10 & 1 & 0 \\
\hline
\end{tabular}

c

\begin{tabular}{|c|c|}
\hline Upregulated genes in Atf7ip KO mESCs (87) & FDR \\
\hline meiotic cell cycle & $1.40 \mathrm{E}-06$ \\
\hline spermatogenesis & 0.001343 \\
\hline synapsis & 0.004172 \\
\hline fertilization & 0.030742 \\
\hline DNA methylation involved in gamete generation & 0.039874 \\
\hline
\end{tabular}

d

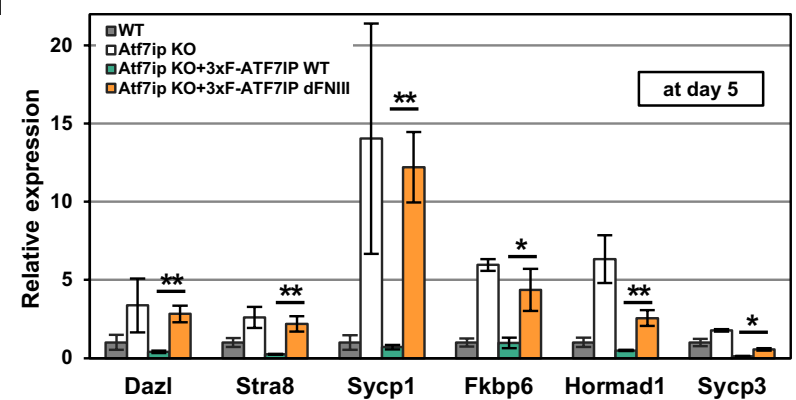

e

Upregulated repeats in Atf7ip KO mESCs

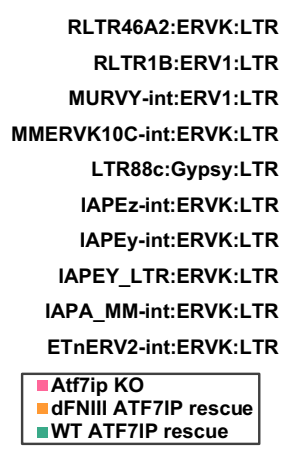

g

\section{ChIP-seq peaks}

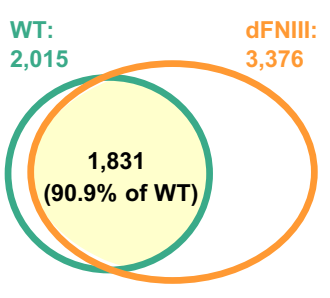

h

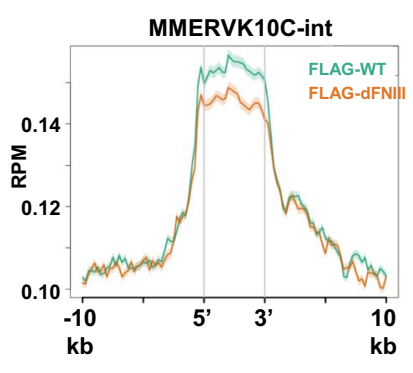

b

Upregulated Genes

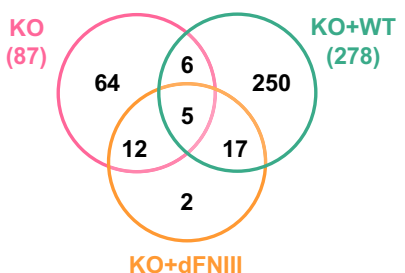

(38)

\begin{tabular}{|c|c|}
\hline FNIII dependent genes (12) & FDR \\
\hline meiotic cell cycle & $2.00 \mathrm{E}-07$ \\
\hline synapsis & $2.31 \mathrm{E}-04$ \\
\hline fertilization & 0.001043 \\
\hline spermatogenesis & 0.016574 \\
\hline synaptonemal complex assembly & 0.036017 \\
\hline
\end{tabular}

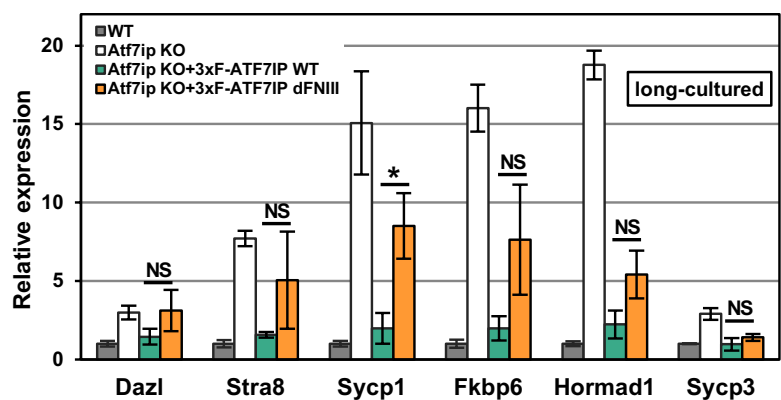

$\mathbf{f}$

Over-suppressed repeats in Atf7ip KO mESCs rescued with ATF7IP WT

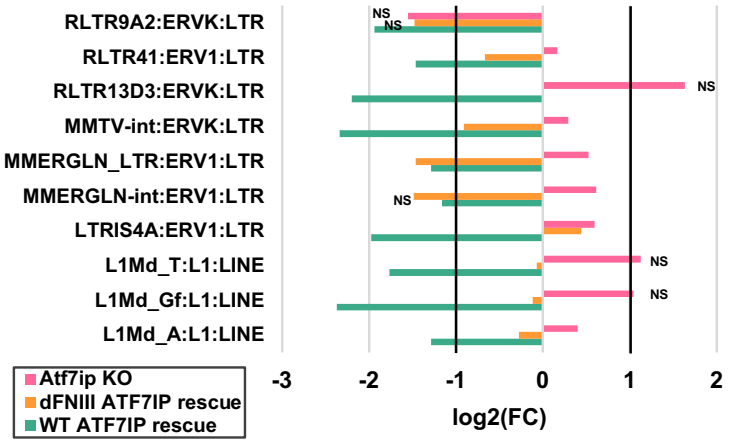

WT ATF7IP rescue
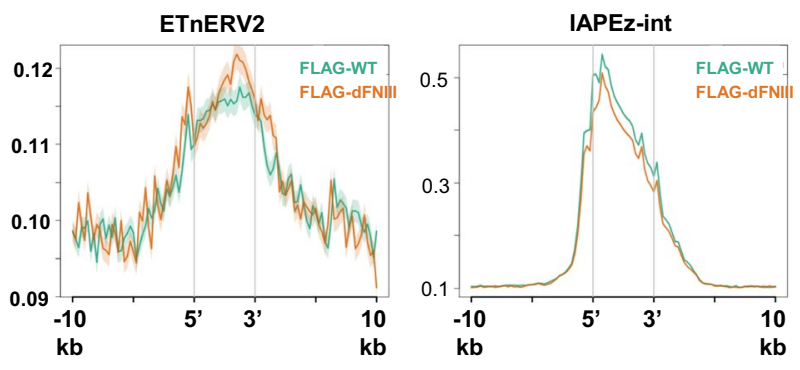
also SETDB1-targeted and de-repressed in Setdb1 KO mESCs $[3,15]$. Since most of the additionally downregulated repeats by exogenous ATF7IP WT expression were indeed weakly (<twofold) de-repressed in Atf7ip $\mathrm{KO}$ ESCs (Fig. 5f), SETDB1-mediated retroelement silencing might be enhanced by the overproduction of ATF7IP WT in mESCs. However, the majority of additionally downregulated repeats by exogenous ATF7IP WT expression were more mildly repressed by the FNIII domain mutant in comparison with ATF7IP WT (Fig. 5f), even though the two molecules were similarly expressed (Fig. 1b), supporting the notion that the FNIII domain of ATF7IP contributes to efficient transcriptional silencing mediated by the SETDB1 complex.

In Zmym2 KO mESCs, multiple genes were also upregulated and downregulated (Fig. 5a). More than half of the upregulated genes (52/87) in Atf7ip KO mESCs were also upregulated in Zmym $2 \mathrm{KO}$ mESCs, whereas a smaller portion of upregulated genes (52/178) in Zmym2 $\mathrm{KO}$ mESCs were also upregulated in Atf7ip KO mESCs (Additional file 9: Table S2 and Additional file 10: Fig. S8A, B), suggesting that the regulation of the majority of the upregulated genes in Zmym2 KO mESCs is independent of FNIII domain interaction. More importantly, however, among the 12 upregulated genes in Atf7ip KO mESCs that were not silenced by the FNIII domain mutant, 11 genes were also upregulated by ZMYM2 depletion (Additional file 9: Table. S2). We confirmed that the genes commonly upregulated in Atf7ip KO mESCs expressing the dFNIII mutant and the Zmym2 $\mathrm{KO}$ mESCs are repressed in the Zmym2 KO mESC complemented with 3xFLAG-ZMYM2 (Additional file 7: Fig. S6C). RNA-seq analysis showed that six different classes of repeats, which were mainly L1 elements, were upregulated in Zmym2 KO mESCs (Fig. 5a and Additional file 10: Fig. S8C). Furthermore, the IAPEy-int retroelement, which was upregulated in Atf7ip KO mESCs and was not silenced by the FNIII domain mutant, was also de-repressed in Zmym2 KO mESCs (Additional file 10: Fig. S8C and D). Thus, ZMYM2 contributes to ATF7IP FNIII domain-dependent transcriptional silencing, including MAX-targeted germ cell-related gene regulation.

Finally, we performed ChIP-seq analysis of Atf7ip KO mESCs rescued with 3xFLAG-tagged WT or the FNIII domain mutant of ATF7IP with an anti-FLAG antibody. We analyzed two samples for each cell type, and commonly detected peaks between two samples were defined as stringent peaks and utilized for subsequent informatics analysis. As shown in Fig. $5 \mathrm{~g}$, more than $90 \%$ of 3xFLAG-ATF7IP WT stringent peaks $(1831 / 2015)$ overlapped with 3xFLAG-dFNIII mutant peaks. Because the number of 3xFLAG-ATF7IP WT peaks for one ChIP-seq sample was about $1 / 3$ that of the other sample (2229 vs. 6331), the stringent peaks of 3xFLAG-ATF7IP WT might be underrepresented. When the total peaks of 3xFLAGATF7IP WT from two ChIP-seq samples were used for the same comparison analysis, $94.1 \%$ of the 3xFLAGdFNIII mutant stringent peaks (3177/3376) overlapped with the 3xFLAG-WT peaks (Additional file 11: Fig. S9A). 3xFLAG-ATF7IP WP was enriched on the transcription start site of some of the upregulated germ cellrelated genes in Atf7ip KO mESCs, but the enrichment of 3xFLAG-dFNIII mutant was lost or diminished on them (Additional file 11: Fig. S9B). We further examined 3xFLAG-ATF7IP accumulation on the retroelements that were major targets of the SETDB1/ATF7IP complex and de-repressed in Atf7ip or Setdb1 KO mESCs. As shown in Fig. 5h, 3xFLAG-ATF7IP WT was enriched in the retroelements and the binding profiles of the 3xFLAG-dFNIII mutant were mostly maintained. These data indicate that the deletion of the FNIII domain does not have a strong impact on ATF7IP targeting and accumulation, especially on SETDB1 target retroelements.

\section{Discussion}

Previous studies have revealed the importance of ATF7IP in transcriptional silencing [11-14]. Considering that ATF7IP is a relatively large protein and contains at least two functional binding surfaces for other proteins (SETDB1 and MBD1), it would be worthwhile to determine the functional requirements of each domain for transcriptional silencing by ATF7IP. We used deletion mutants of ATF7IP and provided evidence for the distinct functional requirements of the two regions of ATF7IP. In our previous study, we showed that ATF7IP plays a pivotal role in the nuclear localization of SETDB1 [16]. Our new study using the dSETDB1 mutant of ATF7IP further indicates that interaction with ATF7IP is essential for ATF7IP-dependent SETDB1 nuclear localization. On the other hand, the dFNIII mutant mostly silenced SETDB1/ ATF7IP-targeted retroelements, but failed to repress some germ cell-related genes (Figs. 1c and $5 \mathrm{c}, \mathrm{d}$ ). To find a clue for the function of the FNIII domain, we performed a proteomic analysis for FNIII-binding proteins and identified over 20 candidate proteins, some of which were validated by independent experiments and possessed a consensus sequence, named FAM (Fig. 3). These FNIIIbinding proteins may be relevant to ATF7IP functions. Among them, ZMYM2, is a member of the MYM-type zinc finger proteins and associates with a transcriptional repressor complex that contains LSD1, CoREST, and HDAC1/2 (LCH complex) via its MYM domain [23, 24]. Although the functions of ZMYM2 in transcriptional regulation have not yet been revealed, it was suggested to contribute to maintain the intact $\mathrm{LCH}$ complex on 
chromatin [24]. Here, we show that ZMYM2 partly mediates transcriptional silencing of the MSCV-GFP reporter integrated into mESCs upon the re-expression of ATF7IP (Fig. 4). ZMYM2 may recruit the $\mathrm{LCH}$ complex to the target loci of ATF7IP and SETDB1 to facilitate the initiation of transcriptional silencing. In addition to ZMYM2, we identified ZMYM4 as an FNIII domain of an ATF7IPbinding protein among other ZMYM family proteins in our proteomic analysis (Additional file 3: Table S1). These proteins may play a role in transcriptional silencing by ATF7IP, as ZMYM2 does. MGA is also potentially related to ATF7IP-mediated silencing of germ cell-related genes. It forms a complex with MAX [2628 ] and plays a role in the silencing of germ cell-related genes in mESCs $[26,29]$. We observed that the dFNIII mutant-rescued cells still showed increased expression of some germ cell-related genes (Fig. 5d). This observation, together with the function of MGA, implies the possibility of the involvement of MGA in ATF7IP-mediated silencing via interaction with the FNIII domain.

\section{Conclusions}

We revealed that the SETDB1-interacting region of ATF7IP is essential for its transcriptional silencing function, but the FNIII domain is mostly dispensable for the silencing of SETDB1/ATF7IP-targeted genes and retroelements. Only some germ cell-related genes expressed in Atf7ip KO ESCs were not silenced by the FNIII domain mutant. The silencing potential of the FNIII domain mutant against SETDB1-targeted retroelements is weaker than that of WT ATF7IP, suggesting that the FNIII domain plays a role as one of the multiple layers in the SETDB1/ATF7IP complex-mediated transcriptional silencing (Fig. 6). We also identified binding proteins for the FNIII domain and their consensus-binding motif.
In addition, we showed that one of the FNIII domainbinding proteins, ZMYM2, partly mediates the efficient silencing of the transgene by ATF7IP. Taken together, this study contributes to the understanding of the function of ATF7IP and its domains.

\section{Methods}

\section{Cell culture and DNA transfection}

mESCs were cultured in Dulbecco's modified Eagle's medium (DMEM, Cat\# D6429; Sigma-Aldrich, USA) supplemented with 15\% KnockOut Serum Replacement (KSR; Cat\# 10828028, Invitrogen, USA), $1 \%$ fetal bovine serum, $0.1 \mathrm{mM} \beta$-mercaptoethanol, leukemiainhibiting factor, and $1 \times$ nonessential amino acids. HEK293T cells were cultured in DMEM (Cat\# 0845755; Nacalai Tesque, Japan) supplemented with $10 \%$ FBS. The mESC lines used in this study as follows: Atf7ip KO (clone name: TT\#2-12), Zmym2 KO (clone name: \#1 and \#2), and Atf7ip/Zmym2 DKO (clone name: \#1 and \#2). Zmym2 KO and Zmym2/Atf7ip DKO cells were established by CRISPR/Cas9 technology using pKLV2U6gRNA5(Bbs1)-PGKpuro2ABFP [30]. Target sequences of gRNAs are listed in Additional file 12: Table S3. Stably transfected cell lines were established by the piggyback transposon system. DNA transfection was performed using Lipofectamine 2000 (Invitrogen, USA; for mESCs) or Polyethylenimine Max (Polyscience, Inc., USA; for HEK293T cells), according to the manufacturer's instructions.

\section{Plasmids}

Full-length mouse ATF7IP expression vector was described previously [22]. The deletion mutants of ATF7IP was generated by PCR-based method. The FNIII domain (residues 1190-1306) cDNA was inserted into pColdGST vector (Takara Bio Ltd., Japan). Full-length

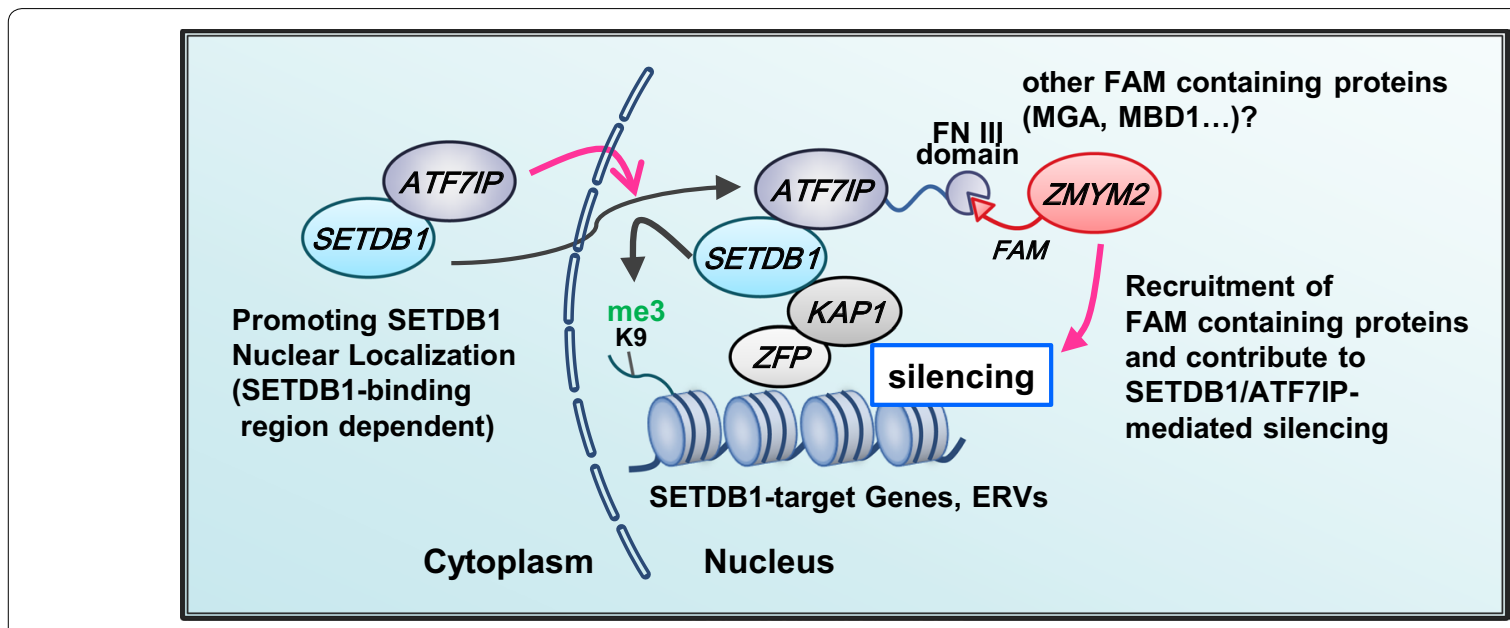

Fig. 6 Proposed model for the role of SETDB1-binding region and FNIII domain of ATF7IP in the SETDB1/ATF7IP complex-mediated silencing 
mouse Zmym2 cDNA was generated from FANTOM clones AK135499 and AK137720. The Zmym2 cDNA was inserted into pPB-CAG-V5-IRES.puromycine vector by In-Fusion technology. Full-length mouse $M b d 1 \mathrm{cDNA}$ was PCR-amplified from FAMTOM clone AK046252 and inserted into pPB-CAG-V5-IRES.puromycine vector by In-Fusion technology. Full-length mouse $Z f p 518 a$ cDNA was PCR-amplified from cDNA of mESCs and inserted into pPB-CAG-3xFLAG-IRES.puromycine vector by In-Fusion technology. Mga 2362-3003, mouse Kiaa1551, and mouse Zmym4 with its $5^{\prime} \mathrm{UTR}$ cDNAs were PCR-amplified from total cDNA of mESCs and inserted into pPB-CAG-V5-IRES.puromycine vector by In-Fusion technology. All the mutant vectors were produced by overlapping PCR with primers harboring mutations. The detailed information of plasmids and the used primers were given in supplemental file (Additional file 9: Table S2).

\section{Purification of recombinant proteins}

Escherichia coli (E. coli) BL21 (pLysS) strains were transformed with expression vectors, and the transformed bacteria were cultured in $2 \times$ YT medium with antibiotics and $0.5 \mathrm{mM}$ isopropyl $\beta$-D-1-thiogalactopyranoside (IPTG) for $16 \mathrm{~h}$ at $16{ }^{\circ} \mathrm{C}$. For purification of GST-tagged proteins, the cultured cell pellets were lysed with lysis buffer ( $1 \mathrm{xPBS} / 0.5 \% \mathrm{NP}-40)$ added with phenylmethanesulfonyl fluoride (PMSF) at $1 \mathrm{mM}$. After sonication with a Branson Sonifier (S-250D, Branson Ultrasonics) for 10 or $20 \mathrm{~min}$ on ice, the lysates were centrifuged at $7300 \times g$ for $10 \mathrm{~min}$. The supernatants were incubated with prewashed Glutathione $4 \mathrm{~B}$ sepharose (GE) for $1-3 \mathrm{~h}$ at $4{ }^{\circ} \mathrm{C}$ with gentle rotation. The beads were washed five times with the lysis buffer and then eluted with elution buffer (50 mM Tris, $10 \mathrm{mM}$ Glutathione, $1 \mathrm{mM}$ dithiothreitol (DTT)). The eluted proteins were dialyzed with $1 \mathrm{xPBS} / 10 \%$ glycerol, and the concentration was measured by the Bradford protein assay and SDS-PAGE.

\section{Western blot and quantitative PCR analysis}

Both analyses were performed as described previously [22]. The antibodies used in this study are described in "Antibodies" section.

The primers used in qPCR analysis are provided in Additional file 9: Table S2.

\section{Immunoprecipitation}

For immunoprecipitation (IP), cells were lysed with normal-lysis buffer $(50 \mathrm{mM}$ Tris- $\mathrm{HCl}$ at $\mathrm{pH} 7.5,150 \mathrm{mM}$ $\mathrm{NaCl}, 1 \mathrm{mM}$ EDTA, $10 \%$ glycerol, 1\% NonidetP-40, $1 \mathrm{mM}$ PMSF, $1 \times$ protease inhibitor cocktail), mid-lysis buffer $(50 \mathrm{mM}$ Tris- $\mathrm{HCl}$ at $\mathrm{pH} 7.5,300 \mathrm{mM} \mathrm{NaCl}$, $1 \mathrm{mM}$ EDTA, $10 \%$ glycerol, $1 \%$ NonidetP-40, $1 \mathrm{mM}$ phenylmethanesulfonyl fluoride (PMSF), $1 \times$ protease inhibitor cocktail; Nacalai Tesque, Japan) or the high-salt lysis buffer $(50 \mathrm{mM}$ Tris- $\mathrm{HCl}$ at $\mathrm{pH} 7.5,500 \mathrm{mM} \mathrm{NaCl}$, $1 \mathrm{mM}$ EDTA, $10 \%$ glycerol, $1 \%$ Nonidet P-40, $1 \mathrm{mM}$ PMSF, $1 \times$ protease inhibitor cocktail; Nacalai Tesque, Japan). After centrifugation at $14,000 \times g$ for $10 \mathrm{~min}$, the supernatants were incubated with anti-FLAG affinity gel (Sigma-Aldrich, USA) or antibody-conjugated Protein A and Protein G Dynabeads mix for at least $3 \mathrm{~h}$ to overnight at $4{ }^{\circ} \mathrm{C}$. The resin was then washed five times with the lysis buffer and eluted by $2 \times$ Laemmli sample buffer. Equivalent amounts of the input and the precipitates were subjected to standard western blot analysis.

\section{Antibodies}

Following antibodies used for this study: anti- $\alpha$-Tubulin (clone B-5-1-2, Sigma-Aldrich); anti-FLAG M2 antibody (F3165, Sigma-Aldrich for western blot and IF assays; F7425, Sigma-Aldrich for IF analysis); antiMCAF1/ATF7IP (ab84497, Abcam); anti-SETDB1/ESET (CP10377, CELL APPLICATIONS, for western blot and IF analyses); anti-V5 (R960-25, Thermo Fisher which is the same as \#46-0705, Life technology); anti-ZMYM2 (A301-711A, Bethyl Laboratories).

\section{Mass spectrometry analysis for identification of the ATF7IP FNIII domain-binding proteins}

mESCs cultured in two $15-\mathrm{cm}$ dishes (approximately $6.0 \times 10^{7}$ cells/dish) were collected, lysed with $8 \mathrm{~mL}$ Buffer A (10 mM HEPES at pH 7.9, $10 \mathrm{mM} \mathrm{KCl}, 1.5 \mathrm{mM}$ $\mathrm{MgCl}_{2}, 0.34 \mathrm{M}$ sucrose, $10 \%$ glycerol, $1 \mathrm{mM}$ DTT, $1 \mathrm{mM}$ PMSF, $1 \times$ protease inhibitor cocktail) containing Triton$\mathrm{X} 100$ at $0.1 \%$ as a final concentration and incubated on ice for $5 \mathrm{~min}$. After centrifugation at $1300 \times g$ for $4 \mathrm{~min}$ at $4{ }^{\circ} \mathrm{C}$, the pellet (nuclear fraction) was re-suspended in $2 \mathrm{~mL}$ normal-lysis buffer and was sonicated. After centrifugation at $14,000 \times g$ for $10 \mathrm{~min}$, the supernatants were incubated with $100 \mu \mathrm{L}$ of Glutathione $4 \mathrm{~B}$ sepharose (50\% slurry) for $1 \mathrm{~h}$. The supernatant was then incubated with the recombinant FNIII domain-immobilized Glutathione $4 \mathrm{~B}$ sepharose ( $2 \mu \mathrm{g}$ protein $/ 10 \mu \mathrm{L}$ beads volume) at $4{ }^{\circ} \mathrm{C}$ overnight. The resin was then washed seven times with the lysis buffer and washed two times additionally with $100 \mathrm{mM}$ ammonium bicarbonate (ABC) buffer. The pellet was re-suspended in $100 \mu \mathrm{L} A B C$ buffer with $1 / 10$ volume of acetonitrile and DTT $(20 \mathrm{mM})$. The mixture was incubated at $56{ }^{\circ} \mathrm{C}$ for $30 \mathrm{~min}$ and then $6 \mu \mathrm{L}$ of $500 \mathrm{mM}$ iodoacetoamide was added and incubated at $37{ }^{\circ} \mathrm{C}$ for $30 \mathrm{~min}$ in the dark. The proteins were digested with $3 \mu \mathrm{g}$ Tripsin/Lys-C mix (Promega) at $37{ }^{\circ} \mathrm{C}$ overnight. The digested protein fragments were applied to quantitative 
MS/MS analysis and following protein identification as described previously [31].

\section{Immunostaining}

Immunostaining was performed as described previously with some minor modifications [32]. mESCs $\left(8.0 \times 10^{4}\right)$ were seeded on 8-well Chamber (192-008, WATSON) which was precoated with $10 \mu \mathrm{g} / \mathrm{mL}$ of laminin for at least $2 \mathrm{~h}$ at $37^{\circ} \mathrm{C}$. After overnight culture, the cells were washed with PBS twice and fixed with $4 \%$ paraformaldehyde for $20 \mathrm{~min}$ at room temperature (RT). After fixation, the cells were permeabilized with $0.2 \%$ Triton X-100 in PBS for $10 \mathrm{~min}$ at RT and were then incubated with $3 \%$ BSA/0.2\% Tween-20 in 4xSSC for 30 min at RT and with primary antibody for additionally $2 \mathrm{~h}$ at RT. After washing twice with $4 \mathrm{xSSC}$, the cells were incubated with secondary antibodies conjugated with Alexa Fluor for $1 \mathrm{~h}$ at RT, washed with $4 \times$ XSSC twice, and finally mounted with ProLong Diamond Antifade Mountant with DAPI (P36961, Thermo Fisher Scientific). Images were obtained using a confocal microscope (FV3000, Olimpus, Japan) and analyzed by Image J (1.50i). The obtained data were further analyzed by R (3.4.1).

\section{ChIP assay}

For crosslinked ChIP (X-ChIP) analysis, $1 \times 10^{7}$ cells were suspended in $1 \mathrm{~mL}$ PBS containing 10\% FBS, and $62.5 \mu \mathrm{L}$ of $16 \%$ formaldehyde (methanol free) was added to the cell suspension. After $10 \mathrm{~min}$ incubation at $25^{\circ} \mathrm{C}$, the cross-linking was quenched by the addition of 100 $\mu \mathrm{L}$ of $2.5 \mathrm{M}$ glycine. After centrifugation at $6500 \times g$ for $3 \mathrm{~min}$ at $4{ }^{\circ} \mathrm{C}$, the pellets were briefly washed with ChIPlysis buffer $(10 \mathrm{mM}$ Tris-HCl, pH 7.5, $10 \mathrm{mM} \mathrm{NaCl}, 0.5 \%$ NP-40, and $1 \times$ protease inhibitor cocktail) and were then incubated with $500 \mu \mathrm{L}$ of the ChIP-lysis buffer for $5 \mathrm{~min}$ on ice. After centrifugation at $6500 \times g$ for $3 \mathrm{~min}$ at $4{ }^{\circ} \mathrm{C}$, the pellets were re-suspended in SDS-lysis buffer (50 mM Tris- $\mathrm{HCl}, \mathrm{pH} 8.0,10 \mathrm{mM}$ EDTA, 1\% SDS, and $1 \times$ protease inhibitor cocktail). After $10 \mathrm{~min}$ incubation on ice, $400 \mu \mathrm{L}$ of Triton buffer $(15 \mathrm{mM}$ Tris- $\mathrm{HCl}$, pH 8.0, $150 \mathrm{mM} \mathrm{NaCl}, 1 \mathrm{mM}$ EDTA, $1 \%$ Triton X-100, and $1 \times$ protease inhibitor cocktail) was added to the suspensions. The cell lysates were then sonicated using a Bioruptor UCD-250 (Diagenode). After centrifugation at $14,000 \times g$ for $10 \mathrm{~min}$ at $4{ }^{\circ} \mathrm{C}$, the supernatants were diluted with Triton buffer to $1100 \mu$. We used 100 $\mu \mathrm{L}$ or $1000 \mu \mathrm{L}$ of the lysates for input samples or IP samples, respectively. For IP, the lysates were incubated with antibody-conjugated magnetic beads (Dynabeads M-280 Sheep antimouse IgG or Dynabeads Protein G, Invitrogen) at $4{ }^{\circ} \mathrm{C}$ for overnight. After sequential washes with low-salt buffer (20 mM Tris-HCl, pH 7.5, $150 \mathrm{mM} \mathrm{NaCl}$, 2 mM EDTA, 1\% Triton X-100, 0.1\% SDS), high-salt buffer (20 mM Tris-HCl, pH 7.5, $500 \mathrm{mM} \mathrm{NaCl}, 2 \mathrm{mM}$ EDTA, 1\% Triton X-100, 0.1\% SDS), LiCl buffer (10 mM Tris-HCl, pH 7.5, 25 mM LiCl, 1 mM EDTA, 1\% Deoxycholate, 1\% NP-40) and TE, the bound DNA was recovered and then analyzed by qPCR.

\section{RNA-seq analysis}

The total RNA was prepared as described above. Sequencing libraries for transcriptome analysis were prepared using TruSeq Stranded mRNA LT Sample Prep Kit (Illumina) and sequenced using HiSeq 2500 (Illumina Inc.). Raw FastQ data were trimmed with Trim Galore (v0.3.7, default parameters) (http://www.bioinformatics. babraham.ac.uk/projects/trim_galore/) and mapped to the mouse GRCm38 genome assembly and UCSC genes database from the UCSC genome browser using TopHat (v2.1.1) [33]. After read mapping, mapped reads were analyzed by TEtranscripts (v1.4.11, default parameters) [34] to calculate gene and repeat expression levels and identify DE genes and repeats (adj. $P$ value $<0.05, \mathrm{FC}>2$ ). UCSC genes database and RepeatMasker track from the UCSC genome browser were used for the calculation of gene and repeat expression levels, respectively.

\section{ChIP-seq analysis}

ChIP-seq libraries were prepared using KAPA Hyper Prep Kit (KAPA Biosystems) and sequenced using HiSeq 2500 (Illumina Inc.). The Raw FastQ data were processed as described above. Processed reads were mapped to the mouse GRCm38 genome assembly using Bowtie (v4.4.6) [35]. Peaks were identified by MACS (v1.4.1) [36]. UCSC genes database and RepeatMasker track from the UCSC genome browser were used for gene and repeat annotations, respectively. Enrichment of ChIP-seq data on retroelement was analyzed by ngsplot v2.47.1 [37].

\section{Supplementary information}

Supplementary information accompanies this paper at https://doi. org/10.1186/s13072-020-00374-4.

Additional file 1: Fig. S1. Related to Fig. 1. A, B Sequence alignment of human and mouse ATF7IP for SETDB1-binding region (A) or FNIII domain (B).

Additional file 2: Fig. S2. Related to Fig. 2. A IF analysis shows that exogenous expression of 3xFLAG-ATF7IP WT and dFNIII mutant rescues the abnormal localization of SETDB1 in Atf7ip KO mESCs at day 5. Representative images are shown, and the quantitative analyses are shown in $B$ and C. Scale bar: $10 \mu \mathrm{m}$. B, C SETDB1 (B) and 3xFLAG-ATF7IP (C) signals in the nucleus that was determined by DAPI staining were calculated. The mean from three independent experiments is shown as a bar graph with jittered points indicating the average \% intensity of each experiment. Over 100 cells were analyzed from a single experiment.

Additional file 3: Table S1. Related to Fig. 3. List of FNIII domain-binding proteins. Proteins highlighted in light blue, light orange, light yellow, and 
light green are the validated proteins, the LCH (LSD1-CoREST-HDAC1) complex, the bait (ATF7IP) and the CPSF (cleavage and polyadenylation specificity factor) complex.

Additional file 4: Fig. S3. Related to Fig. 3. STRING analysis (https://strin $\mathrm{g}$-db.org/) reveals several protein-protein interaction networks in the identified top 20 proteins. LCH complex components: Kdm1a, Rcor2, Hdac1 and Hdac2, CPSF complex components: Cpsf2 and Cstf2t.

Additional file 5: Fig. S4. Related to Fig. 3. A Schematic of ZMYM2 protein and a series of its mutants. The residues $182-350$ was predicted as the region important for the interaction with ATF7IP. Mym domains were shown to interact with LSD1-HDAC complex. B-D Co-IP experiments using each ZMYM2 mutants.

Additional file 6: Fig. S5. Related to Fig. 3. A Co-IP experiment in HEK293T cells shows that mutations at the FAM impede the interaction of MGA C-terminus with ATF7IP. B Co-IP experiment in HEK293T cells shows that mutations at or deletion of the FAM impede the interaction of ZFP518A with ATF7IP. C Co-IP experiment in HEK293T cells shows that mutations at the FAM impede the interaction of ZMYM4 with ATF7IP.

Additional file 7: Fig. S6. Related to Fig. 4. A The transfection of Zmym2 gRNA-expressing vector resulted in a slight increase in the expression of MSCV-GFP reporter, as evidenced by flow cytometric analysis. The Atf7ip gRNA-expressing vector was used as a positive control. B The established Zmym2 KO cell lines show no increase in the expression of MSCV-GFP reporter, as evidenced by flow cytometric analysis. Atf7ip KO mESCs were used as a positive control. C Re-expression of 3xFLAG-ZMYM2 in Zmym2 $\mathrm{KO}$ mESCs repressed the genes upregulated commonly in Atf7ip KO mESCs expressing the dFNIII mutant and the Zmym2 KO mESCs (Fkbp6, Mael and Rec114) or specific in Zmym2 KO mESCs (1700019A02Rik). The data is representative of reproducible results of multiple experiments. Data are mean \pm SEM; NS: $P>0.05,{ }^{*} P<0.05,{ }^{* *} P<0.001$ by Tukey's test.

Additional file 8: Fig. S7. Related to Fig. 5. A The number of replicates of RNA-seq and ChIP-seq. B Correlation between replicates. Scatter plot of $\log 2(\mathrm{RPM}+1)$ of genes between replicate. The figure was generated using the smoothScatter function in R software version 3.5.1. C PCA of RNA-seq data. Principal component analysis (PCA) of gene expression among RNA-seq libraries. The first two components, $P C 1$ and $P C 2$, define the $x$ - and $y$-axes of the two-dimensional space, respectively. The distance between two points reflects the variance in gene expression between them. PC1 and PC2 accounted for $35.9 \%$ and $16.93 \%$, respectively, of the contribution to the variance. PCA was calculated using the prcomp function in R software version 3.5.1.

Additional file 9: Table S2. Related to Fig. 5. List of DE genes and repeats in Atf7ip KO, Atf7ip KO rescued with WT or the FNIII domain mutant of ATF7IP and Zmym2 KO ESCs.

Additional file 10: Fig. S8. Related to Fig. 5. A Overlap of upregulated genes in Atf7ip KO and Zmym2 KO ESCs. B Genes related to gametogenesis or meiosis are commonly derepressed in Atf7ip or Zmym2 KO ESCs. C ATF7IP mainly represses ERV and Zmym2 mainly represses L1. D All retroelement except for IAPEy-int upregulated in Atf7ip KO mESCs are repressed by FN3 independent manner.

Additional file 11: Fig. S9. Related to Fig. 5. A Overlap of FLAG-ATF7IP WT peaks (replicate $1+2$ ) and FNIII domain mutant stringent peaks. B IGV screenshots of RNA-seq and FLAG-ATF7IP ChIP-seq peaks of representative ATF7IP target genes (Dazl and Fkbp6).

Additional file 12: Table S3. Primers and plasmids.

\section{Acknowledgements}

We thank the staff of the Support Unit for Bio-Material Analysis (BMA) at RIKEN Center for Brain Science (CBS) Research Resources Division (RRD) for DNA sequencing, Fantom 3 clone distribution, technical help with LC-MS/MS analysis, and flow cytometric analysis and cell sorting with special thanks to K. Ohtawa.

\section{Authors' contributions}

TT, MK, and YS conceived and designed experiments. MK initiated this study and obtained some important preliminary data. All experiments presented in the paper were performed and analyzed by TT. TT wrote the draft of the paper. MK and YS edited the paper. All authors read and approved the final manuscript.

\section{Funding}

This research was supported by KAKENHI (26250039, 18H03991, and 18H05530) and a RIKEN internal research fund for Y. Shinkai. T.T. was supported by the RIKEN Junior Research Associate Program.

\section{Availability of data and materials}

The ChIP-seq and RNA-seq data have been deposited in the National Center for Biotechnology Information Sequence Read Archive under accession numbers PRJNA664286. Other datasets used and analyzed during the current study are available from the corresponding author on reasonable request.

\section{Ethics approval and consent to participate}

Not applicable.

\section{Consent for publication}

Not applicable.

\section{Competing interests}

The authors declare that they have no competing interests.

\section{Author details}

${ }^{1}$ Cellular Memory Laboratory, RIKEN Cluster for Pioneering Research, Wako 351-0198, Japan. ${ }^{2}$ Present Address: Eli and Edythe Broad Center for Regenerative Medicine and Stem Cell Research, Department of Orthopedic Surgery, University of California, San Francisco, San Francisco, CA, USA.

${ }^{3}$ Present Address: Laboratory for Transcriptome Technology, RIKEN Center for Integrative Medical Sciences (IMS), Yokohama 230-0045, Japan.

Received: 17 July 2020 Accepted: 13 November 2020

Published online: 30 November 2020

\section{References}

1. Mozzetta C, Boyarchuk E, Pontis J, Ait-Si-Ali S. Sound of silence: the properties and functions of repressive Lys methyltransferases. Nat Rev Mol Cell Biol. 2015;16:499-513.

2. Matsui T, Leung D, Miyashita H, Maksakova IA, Miyachi H, Kimura H, Tachibana M, Lorincz MC, Shinkai Y. Proviral silencing in embryonic stem cells requires the histone methyltransferase ESET. Nature. 2010;464:927-31.

3. Karimi MM, Goyal P, Maksakova IA, Bilenky M, Leung D, Tang JX, Shinkai Y, Mager DL, Jones S, Hirst M, et al. DNA methylation and SETDB1/H3K9me3 regulate predominantly distinct sets of genes, retroelements, and chimeric transcripts in mESCs. Cell Stem Cell. 2011;8:676-87.

4. Schlesinger S, Lee AH, Wang GZ, Green L, Goff SP. Proviral silencing in embryonic cells is regulated by Yin Yang 1. Cell Rep. 2013;4:50-8.

5. Wolf G, Yang P, Fuchtbauer AC, Fuchtbauer EM, Silva AM, Park C, Wu W, Nielsen AL, Pedersen FS, Macfarlan TS. The KRAB zinc finger protein ZFP809 is required to initiate epigenetic silencing of endogenous retroviruses. Genes Dev. 2015;29:538-54.

6. Rowe HM, Jakobsson J, Mesnard D, Rougemont J, Reynard S, Aktas T, Maillard PV, Layard-Liesching H, Verp S, Marquis J, et al. KAP1 controls endogenous retroviruses in embryonic stem cells. Nature. 2010;463:237-40.

7. Tchasovnikarova IA, Timms RT, Matheson NJ, Wals K, Antrobus R, Gottgens B, Dougan G, Dawson MA, Lehner PJ. GENE SILENCING. Epigenetic silencing by the HUSH complex mediates position-effect variegation in human cells. Science. 2015;348:1481-5.

8. Wang H, An W, Cao R, Xia L, Erdjument-Bromage H, Chatton B, Tempst P, Roeder RG, Zhang Y. mAM facilitates conversion by ESET of dimethyl to trimethyl lysine 9 of histone $\mathrm{H} 3$ to cause transcriptional repression. Mol Cell. 2003;12:475-87.

9. Ichimura T, Watanabe S, Sakamoto Y, Aoto T, Fujita N, Nakao M. Transcriptional repression and heterochromatin formation by MBD1 and MCAF/ AM family proteins. J Biol Chem. 2005;280:13928-35. 
10. Koch CM, Honemann-Capito M, Egger-Adam D, Wodarz A. Windei, the Drosophila homolog of mAM/MCAF1, is an essential cofactor of the H3K9 methyl transferase dSETDB1/Eggless in germ line development. PLoS Genet. 2009;5:e1000644.

11. Minkovsky A, Sahakyan A, Rankin-Gee E, Bonora G, Patel S, Plath K. The Mbd1-Atf7ip-Setdb1 pathway contributes to the maintenance of $X$ chromosome inactivation. Epigenet Chromatin. 2014;7:12.

12. Matsumura $Y$, Nakaki R, Inagaki T, Yoshida A, Kano Y, Kimura H, Tanaka T, Tsutsumi S, Nakao M, Doi T, et al. H3K4/H3K9me3 bivalent chromatin domains targeted by lineage-specific DNA methylation pauses adipocyte differentiation. Mol Cell. 2015;60:584-96.

13. Thompson PJ, Dulberg V, Moon KM, Foster $\sqcup$, Chen C, Karimi MM, Lorincz MC. hnRNP K coordinates transcriptional silencing by SETDB1 in embryonic stem cells. PLoS Genet. 2015;11:e1004933.

14. Timms RT, Tchasovnikarova IA, Antrobus R, Dougan G, Lehner PJ. ATF7IPMediated stabilization of the histone methyltransferase SETDB1 is essential for heterochromatin formation by the HUSH complex. Cell Rep. 2016;17:653-9.

15. Fukuda K, Okuda A, Yusa K, Shinkai Y. A CRISPR knockout screen identifies SETDB1-target retroelement silencing factors in embryonic stem cells. Genome Res. 2018;28:846-58.

16. Tsusaka T, Shimura C, Shinkai Y. ATF7IP regulates SETDB1 nuclear localization and increases its ubiquitination. EMBO Rep. 2019;20:e48297.

17. Thompson PJ, Macfarlan TS, Lorincz MC. Long terminal repeats: from parasitic elements to building blocks of the transcriptional regulatory repertoire. Mol Cell. 2016;62:766-76.

18. Fujita N, Watanabe S, Ichimura T, Ohkuma Y, Chiba T, Saya H, Nakao M. MCAF mediates MBD1-dependent transcriptional repression. Mol Cell Biol. 2003;23:2834-43.

19. $\mathrm{Ng} \mathrm{HH}$, Jeppesen $\mathrm{P}$, Bird A. Active repression of methylated genes by the chromosomal protein MBD1. Mol Cell Biol. 2000;20:1394-406.

20. Fujita N, Takebayashi S, Okumura K, Kudo S, Chiba T, Saya H, Nakao M. Methylation-mediated transcriptional silencing in euchromatin by methyl-CpG binding protein MBD1 isoforms. Mol Cell Biol. 1999;19:6415-26.

21. Fujita N, Shimotake N, Ohki I, Chiba T, Saya H, Shirakawa M, Nakao M. Mechanism of transcriptional regulation by methyl-CpG binding protein MBD1. Mol Cell Biol. 2000;20:5107-18.

22. Tsusaka T, Kikuchi M, Shimazu T, Suzuki T, Sohtome Y, Akakabe M, Sodeoka M, Dohmae N, Umehara T, Shinkai Y. Tri-methylation of ATF7IP by G9a/GLP recruits the chromodomain protein MPP8. Epigenet Chromatin. 2018;11:56.

23. Hakimi MA, Dong Y, Lane WS, Speicher DW, Shiekhattar R. A candidate $X$-linked mental retardation gene is a component of a new family of histone deacetylase-containing complexes. J Biol Chem. 2003;278:7234-9.

24. Gocke CB, Yu H. ZNF198 stabilizes the LSD1-CoREST-HDAC1 complex on chromatin through its MYM-type zinc fingers. PLoS ONE. 2008;3:e3255.
25. da Huang W, Sherman BT, Lempicki RA. Systematic and integrative analysis of large gene lists using DAVID bioinformatics resources. Nat Protoc. 2009;4:44-57.

26. Maeda I, Okamura D, Tokitake Y, Ikeda M, Kawaguchi H, Mise N, Abe K, Noce T, Okuda A, Matsui Y. Max is a repressor of germ cell-related gene expression in mouse embryonic stem cells. Nat Commun. 2013:4:1754.

27. Hurlin PJ, Steingrimsson E, Copeland NG, Jenkins NA, Eisenman RN. Mga, a dual-specificity transcription factor that interacts with Max and contains a T-domain DNA-binding motif. EMBO J. 1999;18:7019-28.

28. Ogawa H, Ishiguro K, Gaubatz S, Livingston DM, Nakatani Y. A complex with chromatin modifiers that occupies E2F- and Myc-responsive genes in G0 cells. Science. 2002;296:1132-6.

29. Endoh M, Endo TA, Shinga J, Hayashi K, Farcas A, Ma KW, Ito S, Sharif J, Endoh T, Onaga N, et al. PCGF6-PRC1 suppresses premature differentiation of mouse embryonic stem cells by regulating germ cell-related genes. eLife. 2017;6:e21064.

30. Tzelepis K, Koike-Yusa H, De Braekeleer E, Li Y, Metzakopian E, Dovey OM, Mupo A, Grinkevich V, Li M, Mazan M, et al. A CRISPR dropout screen identifies genetic vulnerabilities and therapeutic targets in acute myeloid leukemia. Cell Rep. 2016;17:1193-205.

31. Shimazu T, Barjau J, Sohtome Y, Sodeoka M, Shinkai Y. Selenium-based S-adenosylmethionine analog reveals the mammalian seven-beta-strand methyltransferase METTL10 to be an EF1A1 lysine methyltransferase. PLoS One 2014;9:e105394.

32. Ferry L, Fournier A, Tsusaka T, Adelmant G, Shimazu T, Matano S, Kirsh O, Amouroux R, Dohmae N, Suzuki T, et al. Methylation of DNA ligase 1 by G9a/GLP recruits UHRF1 to replicating DNA and regulates DNA methylation. Mol Cell. 2017;67:550-565.e555.

33. Trapnell C, Pachter L, Salzberg SL. TopHat: discovering splice junctions with RNA-Seq. Bioinformatics. 2009;25:1105-11.

34. Jin Y, Tam OH, Paniagua E, Hammell M. TEtranscripts: a package for including transposable elements in differential expression analysis of RNA-seq datasets. Bioinformatics. 2015;31:3593-9.

35. Langmead B, Trapnell C, Pop M, Salzberg SL. Ultrafast and memoryefficient alignment of short DNA sequences to the human genome. Genome Biol. 2009;10:R25.

36. Zhang Y, Liu T, Meyer CA, Eeckhoute J, Johnson DS, Bernstein BE, Nusbaum C, Myers RM, Brown M, Li W, et al. Model-based analysis of ChIP-Seq (MACS). Genome Biol. 2008;9:R137.

37. Shen L, Shao N, Liu X, Nestler E. ngs.plot: quick mining and visualization of next-generation sequencing data by integrating genomic databases. BMC Genomics. 2014;15:284.

\section{Publisher's Note}

Springer Nature remains neutral with regard to jurisdictional claims in published maps and institutional affiliations.
Ready to submit your research? Choose BMC and benefit from:

- fast, convenient online submission

- thorough peer review by experienced researchers in your field

- rapid publication on acceptance

- support for research data, including large and complex data types

- gold Open Access which fosters wider collaboration and increased citations

- maximum visibility for your research: over $100 \mathrm{M}$ website views per year

At BMC, research is always in progress.

Learn more biomedcentral.com/submissions 